XI International Conference on Structural Dynamics M. Papadrakakis, M. Fragiadakis, C. Papadimitriou (eds.) Athens, Greece, 22-24 June 2020

\title{
A STOCHASTIC AND CONTINUOUS MODEL OF AEOLIAN VIBRATIONS OF CONDUCTORS EQUIPPED WITH STOCKBRIDGE DAMPERS
}

\author{
Francesco Foti ${ }^{1}, V_{\text {incent Denoël }}^{2}$, Luca Martinelli ${ }^{1}$, and Federico Perotti ${ }^{1}$ \\ ${ }^{1}$ Department of Civil and Environmental Engineering, Politecnico di Milano, Italy \\ e-mail: francesco.foti@polimi.it, luca.martinelli@polimi.it, federico.perotti@polimi.it \\ 2 Structural \& Stochastic Dynamics, University of Liège, Belgium \\ Allée de la Découverte 1, 4000 Liège, Belgium \\ email: v.denoel@uliege.be
}

Keywords: Aeolian vibrations, Cable dynamics, Overhead power lines, Stockbridge dampers.

\begin{abstract}
Suspended conductors and guard wires of overhead electrical transmission lines (OHL) are prone to aeolian vibrations, resulting from the alternate shedding of vortices in the wake of the cable. These vibrations can occur under light to moderate wind and, whenever not properly controlled, can induce wear damage and fatigue failures of the cables. Nonlinear passive control devices such as Stockbridge dampers, hence, are often installed along the line spans to reduce the vibration severity. The technical approach to the assessment of aeolian vibrations is based on the Energy Balance Method (EBM) and relies on the simplifying assumption of mono-modal oscillations. Typical aeolian vibration records, however, clearly show that several modes can be simultaneously excited due to wind variations in time and along the span. Aiming at overcoming the mono-modal vibration assumption of the EBM, in the present paper wind forces are modeled as a narrow band stochastic process, centered around the Strouhal frequency of the conductor and with arbitrary cross-correlation in space. A new approach, based on the well-known smooth endochronic Bouc-Wen model, is developed to describe the hysteretic dynamic response of Stockbridge dampers. An iterative solution strategy based on a stochastically equivalent linear damper model is then developed to investigate aeolian vibrations of a suspended cable with a Stockbridge damper attached along its length.
\end{abstract}




\section{INTRODUCTION}

Suspended conductors and guard wires of overhead electrical transmission lines (OHL) are prone to aeolian vibrations, resulting from the alternate shedding of vortices in the wake of the cable. These vibrations can occur under light to moderate wind and are characterized by small-amplitude (typically less than one diameter) transverse oscillations in the cross-wind direction. Vibration frequencies can be in the broad range of 3-200 Hz, depending on the geometry and axial load of the cable (see e.g. [3, 10, 11, 17]). Whenever not properly controlled, aeolian vibrations can induce wear damage and fatigue failures of the cables (e.g. [11]). Nonlinear passive control devices such as Stockbridge dampers, hence, are often installed along the line spans to reduce the vibration severity (e.g. [9]).

The technical approach currently adopted to assess the aeolian vibration level is based on the Energy Balance Method (EBM) and relies on the simplifying assumption of monomodal oscillations (see e.g. [13]). Typical aeolian vibration records, however, clearly show that several modes can be simultaneously excited due to wind variations in time and along the span [5]. Aiming at overcoming the mono-modal vibration assumption of the EBM, Hagedorn and coworkers [15, 16] developed a promising analytical model to evaluate aeolian vibrations of overhead electrical transmission lines within a probabilistic framework. Wind forces were modeled as a narrow band stochastic process, centered around the Strouhal frequency of the conductor and with arbitrary cross-correlation in space. Aeolian vibrations of damped conductors, then, were rigorously studied under the simplifying assumption of linear behavior of the attached passive control devices.

Stockbridge dampers, however, are characterized by a markedly hysteretic dynamic behavior [28, 27]. In the present paper, the formulation proposed by Hagedorn and coworkers is extended to fully account for the nonlinearities related to the damper mechanical response. A new approach, based on the well-known smooth endochronic Bouc-Wen model [23, 6], is first developed to describe the hysteretic dynamic response of Stockbridge dampers. An iterative solution strategy based on a stochastically equivalent linear damper model is then developed to investigate aeolian vibrations of a suspended cable with a Stockbridge damper attached along its length. Finally, the main features and the potential drawbacks of the proposed formulation are illustrated with reference to a simple yet meaningful benchmark case.

\section{FORMULATION OF THE PROBLEM}

\subsection{The cable model}

Let us consider a cable suspended to horizontal supports and with a Stockbridge damper attached at a distance $x_{d}$ from its left end-section (see Figure 1). The cable is subject to the combined action of a constant axial force $H$ and a dynamic load $f=f(x, t)$, where $x$ is a coordinate spanning the free length $l$ of the cable and $t$ is the time. Consistently with classic approximate "externally forced models" for VIV of bluff bodies (see e.g. [25]), the force $f$ is herein introduced to describe the fluctuating lift force induced by the alternate shedding of vortices in the wake of the cable. By neglecting both sagextensibility effects and bending stiffness terms, small planar vibrations are governed by the linear partial differential equation:

$$
-H \partial_{x}^{2} w+\beta \partial_{t} w+\gamma \partial_{t}^{2} w=f-F_{d} \delta\left(x-x_{d}\right), \quad x, x_{d} \in(0, l)
$$




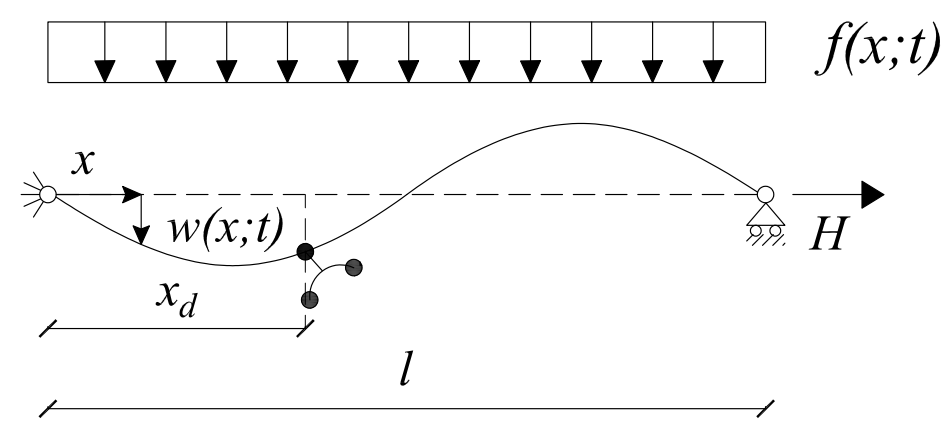

Figure 1: Schematic representation of a suspended cable with length $l$ subject to a constant axial force $H$ and a fluctuating lift force $f(x, t)$. A passive vibration control device is attached at $x=x_{d}$.

where $w=w(x, t)$ is the transverse displacement of the cable centerline, $\gamma$ is the mass per unit of length, $\beta$ is a viscous damping coefficient, $F_{d}$ is the force exerted by the damper on the cable and the symbol $\delta(\cdot)$ denotes the delta Dirac's function. It is worth noting that the herein adopted viscous damping model is frequently adopted in practical applications and research works, in spite of not being fully compliant with experimental evidence and theoretical arguments (see e.g. [7, 8]). This is felt as a possible drawback of the proposed formulation that will be dealt with in the future.

By neglecting the flexibility of the cable supports, Eq. (1) can be solved for prescribed initial conditions under the homogeneous boundary conditions:

$$
w(x=0, t)=w(x=l, t)=0, \quad \forall t
$$

Consistently with the previously stated modeling assumptions, the distributed external force acting on the cable can be defined as:

$$
f(x, t)=\frac{1}{2} \rho d U^{2} q(x, t)
$$

where $\rho$ is the air density, $d$ is the cable diameter, $U$ is the mean wind velocity and $q=q(x, t)$ can be regarded as a space- and time-variable lift coefficient. The lift coefficient can be conveniently modeled (see e.g. [29, 15, 16]) as a stationary stochastic field process with correlation function:

$$
R\left(x_{1}, x_{2}, t\right)=\Psi\left(x_{1}, x_{2}\right) k(t)
$$

where $\Psi\left(x_{1}, x_{2}\right)$ and $k(t)$ are, respectively, a space correlation function and the autocorrelation function of a narrow-band process, centered around the Strouhal frequency 
$\Omega_{s}$, with uni-lateral power spectral density:

$$
G_{q}(\omega)=\frac{1}{2 \pi} \int_{0}^{+\infty} k(t) \exp (-j \omega t) d t=\frac{C_{L, r m s}^{2}}{B \Omega_{s} \sqrt{\pi}} \exp \left[-\left(\frac{1-\frac{\omega}{\Omega_{s}}}{B}\right)^{2}\right], \quad \omega \in \mathbb{R}^{+}
$$

where $C_{L, r m s}$ is the root mean square (r.m.s.) value of the lift coefficient (cf. [29]), and $B$ is a parameter controlling the bandwidth of the spectral peak. By denoting as $S_{t}$ the Strouhal number of the cable, the frequency $\Omega_{s}$ reads:

$$
\Omega_{s}=2 \pi \frac{S_{t} U}{d}
$$

The Strouhal number can be assumed equal to $S_{t}=0.185$ for Reynolds numbers and wind conditions typical of overhead electrical line (OHL) conductors [10, 3]. On the other hand, reference values of the r.m.s. lift coefficient $C_{L, r m s}$ and the bandwidth parameter $B$ typical of OHL conductors, are site-dependent and have not been explicitly reported in the literature, to the authors' knowledge. They could be, however, obtained through in-situ experimental campaigns (e.g. [5]) or wind tunnel tests on scaled models (e.g. [29]). An aperçu of the expected order of magnitude of the model parameters $C_{L, r m s}$ and $B$ can be obtained by looking at the values reported by Vickery and Clark [29] for a slightly tapered cylindrical stack model: $C_{L, r m s}=0.2$ and $B=0.08-0.32$, where the smaller value is associated to smooth flow conditions and the larger one to a turbulence intensity equal to about $10 \%$.

Both Hagedorn and coworkers [15, 16] and Vickery and Clark [29] adopt an exponentially decaying space correlation function $\Psi\left(x_{1}, x_{2}\right)$ (see Eq. (4)). Hagedorn and coworkers introduce the following definition:

$$
\Psi=\Psi_{H}\left(x_{1}, x_{2}\right)=\psi\left(x_{1}\right) \psi\left(x_{2}\right) \exp \left[-\left(\frac{x_{1}-x_{2}}{\delta_{x}}\right)^{2}\right]
$$

without providing, however, experimental information on both the function $\psi(x)$ and the space correlation coefficient $\delta_{x}$. Based on the fitting of experimental measurements on a tapered cylindrical stack model, Vickery and Clark [29] propose the following definition:

$$
\Psi=\Psi_{V C}\left(\bar{r}\left(x_{1}, x_{2}\right)\right)=\cos \left(\frac{\eta_{2}}{\eta_{1}} \bar{r}\right) \exp \left[-\left(\frac{\bar{r}}{\eta_{1}}\right)^{2}\right]
$$

where $\eta_{1}=3, \eta_{2}=2$ and $\bar{r}$ is the non-dimensional distance between two points of the cable identified by the coordinates $x_{1}$ and $x_{2}$ :

$$
\bar{r}=\frac{\left|x_{1}-x_{2}\right|}{d}
$$

In the absence of experimental information explicitly referred to OHL conductors, the space correlation function proposed by Vickery and Clark [29] will also be adopted in the present work to model the lift forces acting on the suspended cable (Eq. (4)).

Figure 2(a) shows the uni-lateral power spectral density of the lift coefficient, $G_{q}(\omega)$, obtained through Eq. (5) under the assumption: $C_{L, r m s}=0.2$. The results are shown for two different values of the bandwidth parameter $B$ (i.e. $B=0.1,0.3$ ) and of the Strouhal 

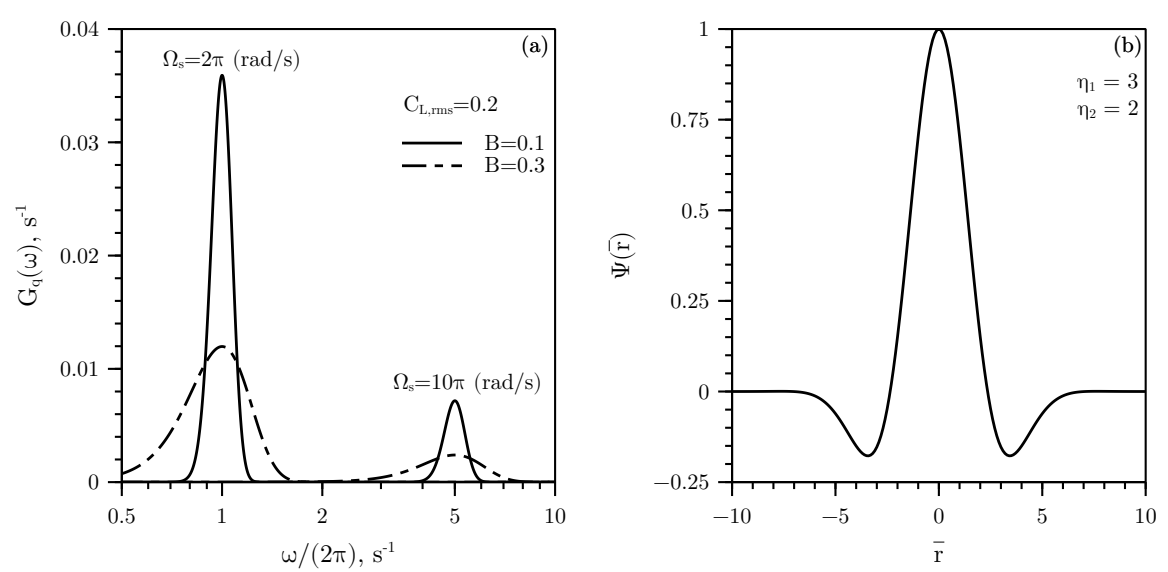

Figure 2: (a) Uni-lateral power spectral density of the lift coefficient, $G_{q}(\omega)$ (see Eq. (5)). The root mean square value of the lift coefficient is assumed equal to $C_{L, r m s}=0.2$. The function $G_{q}(\omega)$ is shown for different values of the bandwidth parameter $B$ (i.e. $B=0.1,0.3$ ) and of the Strouhal frequency (i.e. $\Omega_{s}=2 \pi, 10 \pi \mathrm{rad} / \mathrm{s}$ ). (b) Space coherence function $\Psi(\bar{r})$ (see Eq. 8). Parameters $\eta_{1}$ and $\eta_{2}$ are respectively set equal to $\eta_{1}=3$ and $\eta_{2}=2$.

frequency (i.e. $\Omega_{s}=2 \pi, 10 \pi \mathrm{rad} / \mathrm{s}$ ). Increasing values of the bandwidth parameter $B$, physically related to increasing values of wind turbulence [29], affect the power spectral density of the lift coefficient yielding lower and broader peaks. It is however worth noting that the total energy content of the function $G_{q}(\omega)$ is not affected by the parameter $B$, since $\int_{-\infty}^{+\infty} G_{q}(\omega) d \omega=C_{L, r m s}^{2}$.

It is also worth noting that, not only the total energy content is insensitive to the intensity of turbulence, but also so is the space correlation function $\Psi(\bar{r})$ (depicted in Figure 2(b)) due to the definition herein adopted. These aspect do not appear fully consistent with experimental evidences (e.g. [7, 8]). A thorough experimental testing campaign would be necessary to further assess the validity of these assumptions.

\subsection{The damper model}

The most common type of Stockbridge dampers is made of a metallic clamp, a short metallic strand, also known as "messenger cable," with two inertial bodies attached at its end-sections [10, 9]. The clamp is rigidly attached to the messenger cable and allows connecting the damping device along the span of the OHL conductor. Whenever the clamp undergoes a vertical translation, the two sides of the messenger cables behave as uncoupled flexible cantilevers with lumped translational and rotational masses attached at their ends. Differently than classic linear tuned mass dampers [4], Stockbridge dampers are characterized by a markedly hysteretic dynamic behavior due to the frictional dissipation mechanisms activated by the bending of the messenger cable [28, 9 .

In the present work, we will focus on the special, but practically meaningful case, of symmetric Stockbridge dampers (see Figure 3) under the following simplifying assumptions: (i) the clamp can only undergo vertical translations $w_{d}(t)=w\left(x=x_{d}, t\right)$, and (ii) the rotational inertia of the rigid bodies attached to the end-sections of the messenger cable is neglected. By denoting as $v=v(t)$ the vertical displacement of the tip of the messenger cable with respect to the clamp, the force $F_{d}$ exerted by the damper on the 


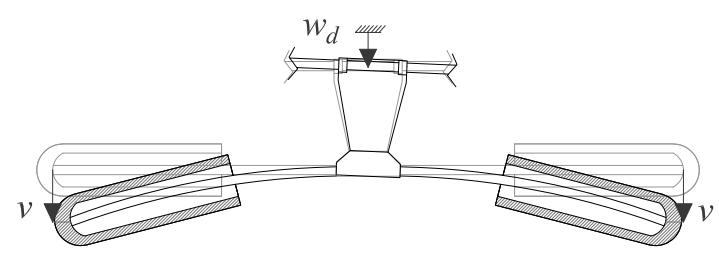

Figure 3: Schematic representation of a symmetric Stockbridge damper. Symbols $w_{d}$ and $v$ denote, respectively, the vertical translation of the clamp and the relative displacement of the tip of the messenger cable with respect to the clamp.

cable (also see Eq.(1)) can be expressed as [9]:

$$
F_{d}=\left(2 m_{d}+m_{c}\right) \ddot{w}_{d}+2 m_{d} \ddot{v}
$$

where $m_{d}$ is the mass of each rigid body attached at the tips of the messenger cable, $m_{c}$ is the mass of the clamp and a dot denotes derivation with respect to time $t$. The mass of the clamp is often significantly smaller than the total mass of the damper and Eq. (10) can be approximately re-written as:

$$
F_{d}=2 m_{d}\left(\ddot{w}_{d}+\ddot{v}\right)
$$

The relative motion of the tip of the messenger cable is governed by the nonlinear ordinary differential equation:

$$
m_{d} \ddot{v}+F_{R}=-m_{d} \ddot{w}_{d}
$$

where $F_{R}$ is the hysteretic restoring force exerted by the messenger cable on the damper mass. Inspired by the work of Pivovarov and Vinogradov [26], a phenomenological modeling approach is adopted to characterize the restoring force $F_{R}$ based on an application of the well known smooth endochronic Bouc-Wen hysteretic model [23, 6]. To this aim, the five-parameter Bouc-Wen model summarized by Ikhouane and coworkers [20, 21, 22] is herein re-stated in a slightly modified form:

$$
\left\{\begin{array}{l}
F_{R}=\alpha k_{d} v+(1-\alpha) k_{d} v_{0} z \\
\dot{z}=\frac{1}{v_{0}}\left[\dot{v}-\sigma|\dot{v}||z|^{n-1} z+(\sigma-1) \dot{v}|z|^{n}\right]
\end{array}\right.
$$

where $z(t) \in[-1,1]$ is a non-dimensional hysteretic variable, $k_{d}>0$ is the initial stiffness of the first-loading branch of the load-displacement curve $F_{R}=F_{R}(v(t), z(t)), \alpha>0$ is the ratio between the minimum theoretical value of the post-yielding tangent stiffness of the of the first-loading branch and $k_{d}, v_{0}$ is a non-negative parameters with the units of a displacement. Finally, $\sigma$ and $n$ are non-dimensional parameters controlling the shape of the hysteresis cycles. It is worth noting that, to ensure the stability and the thermodynamic admissibility of the Bouc-Wen model (13), the parameters $\sigma$ and $n$ should satisfy 
the following inequalities: $\sigma \geq 1 / 2$ and $n \geq 1$ [22]. In order to avoid cumbersome calculations and to keep the model as simple as possible, in the present work the parameters $\sigma$ and $n$ are both set equal to the unity and the second Eq. in 13 is re-written as:

$$
\dot{z}=\frac{1}{v_{0}}(\dot{v}-|\dot{v}| z)
$$

\subsection{Non-dimensional formulation}

In the following, the governing equations of the problem introduced in Sections 2.1 and 2.2 will be re-stated in a more convenient non-dimensional form. To this aim, let us introduce the non-dimensional space $(\xi)$ and time $(\tau)$ variables:

$$
\xi=\frac{x}{l} \in[0,1], \quad \tau=\Omega_{c} t \in \mathbb{R}^{+}
$$

where $\Omega_{c}$ is the characteristic frequency of the cable:

$$
\Omega_{c}=\sqrt{\frac{H}{\gamma l^{2}}}
$$

Substitution of Eqs. (15) and (16) in Eq. (1) yields the non-dimensional equation of motion:

$$
-\partial_{\xi}^{2} \bar{w}+2 \zeta_{c} \partial_{\tau} \bar{w}+\partial_{\tau}^{2} \bar{w}=\bar{f}-\bar{F}_{d} \delta\left(\xi-\xi_{d}\right), \quad \xi, \xi_{d} \in(0,1)
$$

where $\bar{w}=\frac{w(x(\xi), t(\tau))}{d}, \zeta_{c}=\frac{l^{2} \Omega_{c}}{2 H} \beta, \bar{f}=\frac{l^{2}}{H d} f(x(\xi), t(\tau))=\frac{\rho l^{2} U^{2}}{2 H} q(x(\xi), t(\tau)), \bar{F}_{d}=\frac{l}{d H} F_{d}$, and $\xi_{d}=\frac{x_{d}}{l}$. The non-dimensional counterpart of the homogeneous boundary conditions (2) reads:

$$
\bar{w}(\xi=0, \tau)=\bar{w}(\xi=1, \tau)=0, \quad \forall \tau
$$

Starting from Eq. 11, the non-dimensional force $\bar{F}_{d}$ exerted by the damper on the cable can be expressed as:

$$
\bar{F}_{d}=\mu\left(\frac{d^{2} \bar{w}_{d}}{d \tau^{2}}+\frac{d^{2} \bar{v}}{d \tau^{2}}\right)
$$

where $\bar{v}=\frac{v(x(\xi), t(\tau))}{d}, \bar{w}_{d}=\frac{w_{d}}{d}=\bar{w}\left(\xi=\xi_{d}, \tau\right)$ and $\mu$ is the mass ratio:

$$
\mu=\frac{2 m_{d}}{\gamma l}
$$

The relative motion of the tip of the messenger cable (see Eqs. (12), (13)-a and (14)), then, is governed by the non-dimensional equations:

$$
\left\{\begin{array}{l}
\frac{d^{2} \bar{v}}{d \tau^{2}}+\alpha \Theta^{2} \bar{v}+(1-\alpha) \Theta^{2} \bar{v}_{0} \bar{z}=-\frac{d^{2} \bar{w}_{d}}{d \tau^{2}} \\
\frac{d \bar{z}}{d \tau}=\frac{1}{\bar{v}_{0}}\left(\frac{d \bar{v}}{d \tau}-\left|\frac{d \bar{v}}{d \tau}\right| \bar{z}\right)
\end{array}\right.
$$

where $\Omega_{d}=\sqrt{\frac{k_{d}}{m_{d}}}, \Theta=\frac{\Omega_{d}}{\Omega_{c}}, \bar{v}_{0}=\frac{v_{0}}{d}$ and $\bar{z}=z(t(\tau))$. 


\section{PROPOSED SOLUTION STRATEGY}

In this Section, the response of a bare suspended cable (i.e. a cable without attached damper) to the stochastic wind model defined in Section 2.1 is first considered (Section 3.1). The solution is then extended to the case of a cable equipped with a linear passive damping device (Section 3.2), by following the same approach proposed by Hagedorn and coworkers [15, 16]. Finally, the solution strategy is generalized to deal with the non-linearities that characterize the Stockbridge damper model presented in Section 2.2.

\subsection{Response of a bare cable}

Let us preliminary consider the response of a bare suspended cable subject to a concentrated force $F_{1}=\frac{d H}{l} \exp (j \omega t)$ applied at the generic abscissa $x=x_{1}$. The nondimensional equation of motion (see Section 2.3), hence, reads:

$$
-\partial_{\xi}^{2} \bar{w}+2 \zeta_{c} \partial_{\tau} \bar{w}+\partial_{\tau}^{2} \bar{w}=\delta\left(\xi-\xi_{1}\right) \exp (j \bar{\omega} \tau), \quad \xi, \xi_{1} \in(0,1), \quad \bar{\omega}=\frac{\omega}{\Omega_{c}}
$$

Stationary oscillatory solutions of Eq. 22 satisfying the homogeneous boundary conditions (18) can be expressed as $\bar{w}(\xi, \tau)=\phi(\xi) \exp (j \bar{\omega} \tau)$, where $\phi$ is the solution of the boundary value problem ("Green's function", see e.g. [18]):

$$
\left\{\begin{array}{l}
-\phi^{\prime \prime}+2 \zeta_{c} j \bar{\omega} \phi-\bar{\omega}^{2} \phi=\delta\left(\xi-\xi_{1}\right) \\
\phi(0)=\phi(1)=0
\end{array}\right.
$$

and an apex denotes derivation with respect to $\xi$.

Solution of (23) is straightforward and can be expressed, by explicitly highlighting its dependence on both $\xi_{1}$ and $\bar{\omega}$,as:

$$
\phi\left(\xi, \xi_{1}, \bar{\omega}\right)= \begin{cases}\frac{\sin [\nu(\bar{\omega}) \xi] \sin \left[\nu(\bar{\omega})\left(1-\xi_{1}\right)\right]}{\nu(\bar{\omega}) \sin [\nu(\bar{\omega})]} & 0 \leq \xi \leq \xi_{1}^{-} \\ \frac{\sin [\nu(\bar{\omega})(1-\xi)] \sin \left[\nu(\bar{\omega}) \xi_{1}\right]}{\nu(\bar{\omega}) \sin [\nu(\bar{\omega})]} & \xi_{1}^{+} \leq \xi \leq 1\end{cases}
$$

with the definition:

$$
\nu=\sqrt{\bar{\omega}^{2}-2 \zeta_{c} j \bar{\omega}}
$$

Let us consider now a bare cable subject to the the stochastic wind model fully detailed in Section 2.1. Knowledge in closed form of the Green function $\phi=\phi\left(\xi, \xi_{1}, \bar{\omega}\right)$ allows one to easily evaluate (see e.g. [24]) the uni-lateral cross power spectral density function $G_{w}\left(x_{1}, x_{2}, \omega\right)$ of the transverse displacements $(w=w(x, t))$ as:

$$
G_{w}\left(x_{1}, x_{2}, \omega\right)=\left(\frac{1}{2} \rho d U^{2}\right)^{2} \frac{l^{4}}{H^{2}} \bar{C}\left(\xi_{1}\left(x_{1}\right), \xi_{2}\left(x_{2}\right), \bar{\omega}(\omega)\right) G_{q}(\omega)
$$

where $G_{q}(\omega)$ is defined in Eq. (5) and $\bar{C}$ is the two-dimensional integral:

$$
\bar{C}\left(\xi_{1}, \xi_{2}, \bar{\omega}\right)=\int_{0}^{1} \int_{0}^{1} \phi^{*}\left(\zeta_{1}, \xi_{2}, \bar{\omega}\right) \phi\left(\xi_{1}, \zeta_{2}, \bar{\omega}\right) \bar{\Psi}\left(\zeta_{1}, \zeta_{2}\right) d \zeta_{1} d \zeta_{2}
$$


where an asterisk is adopted to denote complex conjugate values. Finally, the variance of the displacement is a function of the arc-length coordinate $x$ and reads:

$$
\sigma_{w}^{2}(x)=\int_{0}^{+\infty} G_{w}(x, x, \omega) d \omega
$$

\subsection{Response of a cable equipped with a linear damper}

By following the same approach adopted in Section 3.2, let us preliminary consider the response of a suspended cable with a damper attached at $x=x_{d}$ and subject to a concentrated force $F_{1}=\frac{d H}{l} \exp (j \omega t)$ applied at the generic abscissa $x=x_{1}$. The non-dimensional equation of motion (see again Section 2.3), hence, reads:

$$
\begin{gathered}
-\partial_{\xi}^{2} \bar{w}+2 \zeta_{c} \partial_{\tau} \bar{w}+\partial_{\tau}^{2} \bar{w}=-\bar{F}_{d} \delta\left(\xi-\xi_{d}\right)+\delta\left(\xi-\xi_{1}\right) \exp (j \bar{\omega} \tau), \\
\xi, \xi_{d}, \xi_{1} \in(0,1), \quad \bar{\omega}=\frac{\omega}{\Omega_{c}}
\end{gathered}
$$

where $\bar{F}_{d}$ is the non-dimensional counterpart of the force exerted by the damper on the cable.

Under the assumption of linear behavior of the attached damper, the non-dimensional force of the damper and clamp displacement can be respectively expressed as:

$$
\bar{F}_{d}(\tau)=\hat{F}_{d} \exp (j \bar{\omega} \tau) \quad \text { and } \quad \bar{w}_{d}(\tau)=\hat{W}_{d} \exp (j \bar{\omega} \tau)
$$

Moreover, the following linear relation can always be introduced:

$$
\hat{F}_{d}=Z_{d, F W}(\bar{\omega}) \hat{W}_{d}
$$

where $Z_{d, F W}=Z_{d, F W}(\bar{\omega})$ is the non-dimensional frequency response function of the linear damper. Substitution of Eqs. (30) and (31) in Eq. (29) yields:

$$
\begin{aligned}
-\partial_{\xi}^{2} \bar{w}+2 \zeta_{c} \partial_{\tau} \bar{w}+\partial_{\tau}^{2} \bar{w}= & {\left[-Z_{d, F W}(\bar{\omega}) \hat{W}_{d} \delta\left(\xi-\xi_{d}\right)+\delta\left(\xi-\xi_{1}\right)\right] \exp (j \bar{\omega} \tau), } \\
& \xi, \xi_{d}, \xi_{1} \in(0,1), \quad \bar{\omega}=\frac{\omega}{\Omega_{c}}
\end{aligned}
$$

Stationary oscillatory solutions of Eq. (32) satisfying the homogeneous boundary conditions 18 can be expressed as $\bar{w}(\xi, \tau)=\phi_{d}(\xi) \exp (j \bar{\omega} \tau)$, where $\phi_{d}$ is the solution of the boundary value problem ("Green's function"):

$$
\left\{\begin{array}{l}
-\phi_{d}^{\prime \prime}+2 \zeta_{c} j \bar{\omega} \phi_{d}-\bar{\omega}^{2} \phi_{d}=-Z_{d, F W}(\bar{\omega}) \hat{W}_{d} \delta\left(\xi-\xi_{d}\right)+\delta\left(\xi-\xi_{1}\right) \\
\phi_{d}(0)=\phi_{d}(1)=0
\end{array}\right.
$$

and an apex denotes derivation with respect to $\xi$. By exploiting the definition of the Green's function $\phi=\phi\left(\xi, \xi_{1}, \bar{\omega}\right)$ introduced in the previous Section (Section 3.1, Eq. (23)) and the superposition principle, it can be easily shown that:

$$
\hat{W}_{d}=\frac{\phi\left(\xi_{d}, \xi_{1}, \bar{\omega}\right)}{1+\phi\left(\xi_{d}, \xi_{d}, \bar{\omega}\right) Z_{d, F W}(\bar{\omega})}
$$


and

$$
\phi_{d}\left(\xi, \xi_{1}, \xi_{d}, \bar{\omega}\right)=\frac{-Z_{d, F W}(\bar{\omega}) \phi\left(\xi_{d}, \xi_{1}, \bar{\omega}\right) \phi\left(\xi, \xi_{d}, \bar{\omega}\right)}{1+\phi\left(\xi_{d}, \xi_{d}, \bar{\omega}\right) Z_{d, F W}(\bar{\omega})}+\phi\left(\xi, \xi_{1}, \bar{\omega}\right)
$$

The response of the suspended cable subject to the stochastic wind model, defined in Section 2.1, then, can be calculated through Eqs. (26)-(28) by simply replacing the Green function $\phi$ with $\phi_{d}$.

\subsection{Response of a cable equipped with a Stockbridge damper}

Let us now consider the case of a suspended cable with a Stockbridge damper attached at $x=x_{d}$. The dynamic behavior of the damper is described through the non-linear model detailed in Section 2.2, based on an application of the Bouc-Wen hysteretic model. The response of the coupled cable+damper system subject to the stochastic wind model defined in Section 2.1 can be sought through an iterative solution strategy based on: (i) the definition of a stochastically equivalent damper model and (ii) the solution strategy already developed in Section 3.2 under the simplifying assumption of linearity of the damper behavior.

The motion of the Stockbridge damper subjected to a prescribed clamp displacement $w_{d}(t)=w\left(x=x_{d}, t\right)$ is governed by the non-dimensional system of Eqs. (21). Under a stochastic loading, the motion of the clamp is a stochastic process with uni-lateral power spectral density $G_{w d}=G_{w d}(\omega)=G_{w}\left(x_{1}=x_{d}, x_{2}=x_{d}, \omega\right)$ (see Eq. 26) and variance $\sigma_{w d}^{2}=\sigma_{w}^{2}\left(x=x_{d}\right)$ (Eq. (28)). A stochastically equivalent linear system of equations describing the motion of the Stockbridge damper, then, can be defined by replacing the second equation in (21) with a linear evolution law for the hysteretic variable $\bar{z}$, i.e.:

$$
\left\{\begin{array}{l}
\frac{d^{2} \bar{v}}{d \tau^{2}}+\alpha \Theta^{2} \bar{v}+(1-\alpha) \Theta^{2} \bar{v}_{0} \bar{z}=-\frac{d^{2} \bar{w}_{d}}{d \tau^{2}} \\
\frac{d \bar{z}}{d \tau}=\frac{1}{\bar{v}_{0}}\left(c_{e} \frac{d \bar{v}}{d \tau}+k_{e} \bar{z}\right)
\end{array}\right.
$$

The linearization coefficients $c_{e}$ and $k_{e}$ can be evaluated in order to minimize the expectation of the squared error $\epsilon^{2}=\left(\frac{d \bar{v}}{d \tau}-\left|\frac{d \bar{v}}{d \tau}\right| \bar{z}-c_{e} \frac{d \bar{v}}{d \tau}-k_{e} \bar{z}\right)^{2}$. By assuming a joint gaussian probability density function for the variables $\left(\bar{v}, \frac{d \bar{v}}{d \tau}, \bar{z}\right)$, the coefficients $c_{e}$ and $k_{e}$ can be evaluated as a special case of the more general expressions proposed by Hurtado and Barbat [19] and read:

$$
\begin{gathered}
c_{e}=1-\frac{2 \sqrt{2}}{\pi} \Gamma\left(\frac{3}{2}\right) \sigma_{z} \rho_{\dot{v} z} \\
k_{e}=-\frac{2 \sqrt{2}}{\pi} \Gamma\left(\frac{3}{2}\right) \sigma_{\dot{v}}
\end{gathered}
$$

where $\sigma_{z}, \sigma_{\dot{v}}$ and $\rho_{\dot{v} z}$ denote the standard deviations and the correlation coefficient of the variables $\bar{v}$ and $\bar{z}$. The variables $\sigma_{z}, \sigma_{\dot{v}}$ and $\rho_{\dot{v} z}$ can be evaluated, for a prescribed motion of the clamp, by solving the linear system of equations (36). By following the approach proposed by Giaralis ([14], Section 6.2.3, pp. 151-157) the following equations can be easily derived:

$$
\sigma_{\dot{v}}^{2}=\int_{0}^{+\infty} A(\bar{\omega}) A^{*}(\bar{\omega}) \bar{\omega}^{6} \frac{\Omega_{c} G_{w d}(\omega(\bar{\omega}))}{d^{2}} d \bar{\omega}
$$




$$
\begin{gathered}
\sigma_{z}^{2}=\int_{0}^{+\infty} B(\bar{\omega}) B^{*}(\bar{\omega}) \bar{\omega}^{4} \frac{\Omega_{c} G_{w d}(\omega(\bar{\omega}))}{d^{2}} d \bar{\omega} \\
\rho_{\dot{v} z}=-\frac{k_{e}}{c_{e}} \cdot \frac{\sigma_{z}}{\sigma_{\dot{v}}}
\end{gathered}
$$

with the definitions:

$$
\begin{aligned}
& A(\bar{\omega})=\frac{\frac{-k_{e}}{\bar{v}_{0}}+j \bar{\omega}}{\left(\alpha \Theta^{2}-\bar{\omega}^{2}\right)\left(\frac{-k_{e}}{\bar{v}_{0}}+j \bar{\omega}\right)+j \bar{\omega}(1-\alpha) \Theta^{2} c_{e}} \\
& B(\bar{\omega})=\frac{j \bar{\omega} c_{e}}{\left(\alpha \Theta^{2}-\bar{\omega}^{2}\right)\left(\frac{-k_{e}}{\bar{v}_{0}}+j \bar{\omega}\right)+j \bar{\omega}(1-\alpha) \Theta^{2} c_{e}}
\end{aligned}
$$

Finally, after some straightforward calculations, herein omitted for the sake of conciseness, the non-dimensional frequency response function of the stochastically equivalent linear damper (see Eq. (31)) can be expressed as:

$$
Z_{d, F W}(\bar{\omega})=-\mu \bar{\omega}^{2}(1+A(\bar{\omega}))
$$

Once $Z_{d, F W}(\bar{\omega})$ is known, a chain substitution into Eqs. (35), (27), 26) and (28) allows one to find a new uni-lateral power spectral density $G_{w d}(\omega)$ and variance $\sigma_{w d}^{2}$. The process is iterated until convergence on $\sigma_{w}^{2}(x)$ is obtained within a prescribed tolerance. This fixed point algorithm is known to be less efficient than a Newton-Raphson approach [2] but has been sufficiently efficient to solve the problem at hand without any big convergence issues.

\section{APPLICATION}

In the following, the proposed modeling procedure is used to investigate the dynamic response of a benchmark OHL span already studied elsewhere (see e.g. [12, 13]). The length of the span is $450 \mathrm{~m}$ and the cable is a ACSR Bersfort $48 / 7$ conductor (diameter $d=35.6 \mathrm{~mm}$, mass per unit of length $\gamma=2.375 \mathrm{~kg} / \mathrm{m}$, Rated Tensile Strength $R T S=$ $180 \mathrm{kN}$ ) subject to an axial force $H=0.2 R T S=36 \mathrm{kN}$. The non-dimensional viscous damping coefficient $\zeta_{c}$, that approximately account for both the aerodynamic and internal damping of the cable, is assumed equal to $\zeta_{c}=0.001$. It is worth noting that, although the order of magnitude of this value seems to be consistent with some experimental data reported in the literature (e.g. [1]), as already noticed in Section 2.1 the adopted viscous damping model is not expected to be fully compliant with all experimental evidences and theoretical arguments of the literature (see e.g. [7, 8]).

A symmetric Stockbridge damper that was experimentally tested by Sauter and Hagedorn [27, 28] is assumed to be applied at the $5 \%$ and $10 \%$ of the span (i.e. at the non-dimensional arc-length coordinate $\xi_{d}=0.05$ and $\left.\xi_{d}=0.10\right)$. The mass attached at the both tips of the messenger cable is equal to $m_{d}=0.856 \mathrm{~kg}$, leading to a mass ratio $\mu=8 \cdot 10^{-4}$ (Eq. (20)).

Sauter and Hagedorn [27, 28] tested the damper on a shaker with sweep tests in frequency at two different constant values of the clamp velocity $\left(\dot{w}_{d}\right)$, namely $0.05 \mathrm{~m} / \mathrm{s}$ and $0.2 \mathrm{~m} / \mathrm{s}$. From these tests the parameter of the proposed Bouc-Wen model of the damper (see Section 2.2) were identified to match the experimental data obtained for the clamp velocity $0.2 \mathrm{~m} / \mathrm{s}$. The identified model parameters are: $k_{d}=8 \mathrm{~N} / \mathrm{mm}, \alpha=0.25$ and 


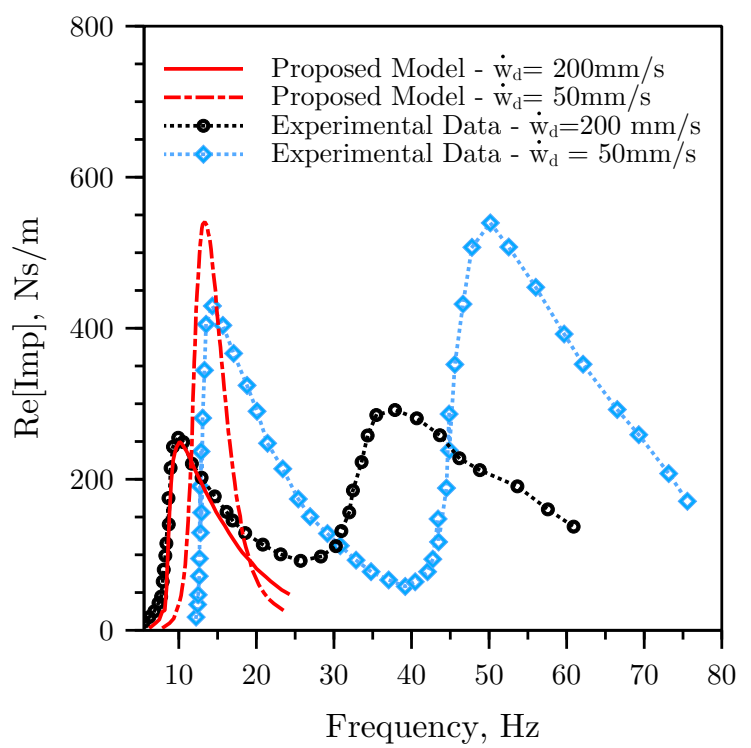

Figure 4: Real part of the impedance curve of the Stockbridge damper tested on a shaker for sweep tests in frequency with an imposed vertical translation of the clamp having a constant value of the clamp velocity equal to $\dot{w}_{d}=0.05 \mathrm{~m} / \mathrm{s}$ and $\dot{w}_{d}=0.2 \mathrm{~m} / \mathrm{s}$. Comparison between the results of the proposed non-linear damper model (see Section 2.2) and experimental data from [27.

$v_{0}=2 \mathrm{~mm}$. The required numerical simulations were performed by applying a sinusoidal motion of the clamp with a frequency undergoing a linear sweep from 0 to $50 \mathrm{~Hz}$ during a total duration of $100 \mathrm{~s}$. The non-linear equations of motion of the damper, then, were numerically integrated at constant time step $\Delta t=10^{-5} \mathrm{~s}$ with a Newmark constant acceleration time-stepping strategy. Iterative corrections were performed through a standard Newton-Raphson scheme at each step.

Figure 4 shows the comparison between experimental results and numerical results in terms of the real part of the impedance function of the damper. It is worth noting that the predictions of the proposed damper model match very well the experimental results not only for the clamp velocity used in the BW identification process $\left(\dot{w}_{d}=0.2 \mathrm{~m} / \mathrm{s}\right)$, but also for $\dot{w}_{d}=0.05 \mathrm{~m} / \mathrm{s}$.

Based on the input data listed above, application of the proposed procedure leads to the results depicted in Figure 5.

Figure 5(a) reports for a line without dampers the standard deviation of the displacement ad the line midspan, expressed as a fraction of the conductor diameter, as a function of the mean wind velocity. Results are reported for two different values of the bandwidth parameter B. Figure 5(b) reports the same quantities for $B=0.1$ and a line with a damper located at 5\% the span (grey broken line), 10\% the span (grey solid line). The results referred to the base cable (black solid line) are also reported to facilitate compartison. From this figure some comments can be drawn. As it can be appreciated, (a) in spite the damper was not optimized, it is nevertheless effective over a wide range of wind velocities; (b) at higher wind velocities, the damper is less effective, as it was expected since the proposed model does not account for the second resonance peak shown in Figure 4 (e.g. at $37 \mathrm{~Hz}$ and $55 \mathrm{~Hz}$ for the $50 \mathrm{~mm} / \mathrm{s}$ and $200 \mathrm{~mm} / \mathrm{s}$ clamp velocity values, respectively). 

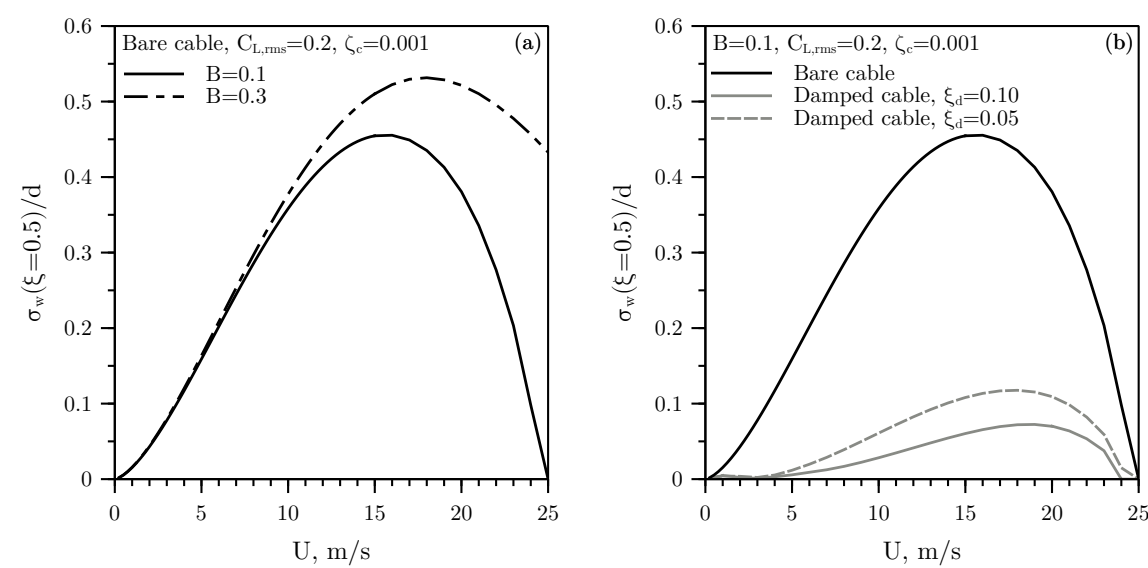

Figure 5: Standard deviation of the cable response at midspan, expressed as a fraction of the cable diameter (a) without attached Stockbridge damper, (b) with attached Stockbridge damper. The curve $B=0.1$ is similar in both plots to facilitate comparison.

\section{CONCLUSIONS}

A stochastic and continuous model of aeolian vibrations of overhead electrical line conductors equipped with Stockbridge dampers has been presented in this paper. Focusing on small-amplitude planar transverse vibrations, suspended cables were modeled through the classic taut-string model. Consistently with classic approximate "externally forced models" for vortex induced vibrations (VIV) of bluff bodies, the wind forces acting on the continuous cable model were described as a narrow band stochastic process, centered around the Strouhal frequency of the conductor and with arbitrary cross-correlation in space. A new approach, based on the well-known smooth endochronic Bouc-Wen model, was developed to describe the hysteretic dynamic response of Stockbridge dampers. An iterative solution strategy based on a stochastically equivalent linear damper model, then, was presented to investigate aeolian vibrations of a suspended cable with a Stockbridge damper attached along its length. Finally, the main features and the potential drawbacks of the proposed formulation were illustrated with reference to a simple yet meaningful benchmark case.

\section{REFERENCES}

[1] D. Brika, A. Laneville, Vortex-induced vibrations of a long flexible circular cylinder, Journal of Fluid Mechanics, 250, 481-508, 1993.

[2] T. Canor, N. Blaise, V. Denoel, An asymptotic expansion-based method for a spectral approach in equivalent statistical linearization, Probabilistic Engineering Mechanics, 38, 1-12, 2014.

[3] CIGRE, In: G. Diana (Ed.), An asymptotic expansion-based method for a spectral approach in equivalent statistical linearization, CIGRE Green Books, Springer, 2018.

[4] J.P. Den Hartog, Mechanical Vibrations, Dover Publications, New York, NY, USA, 14th edition, 1985. 
[5] V. Denoel, T. Andrianne, Real-scale observations of vortex induced vibrations of stay-cables in the boundary layer, Procedia Engineering, 199, 3109-3114, 2017.

[6] M. Domaneschi, Simulation of controlled hysteresis by the semi-active Bouc-Wen model, Computers \& Structures, 106-107, 245-257, 2012.

[7] F. Foti, L. Martinelli, A unified analytical model for the self-damping of stranded cables under aeolian vibrations, Journal of Wind Engineering 8 Industrial Aerodynamics, 176, 225-238, 2018.

[8] F. Foti, L. Martinelli, An enhanced unified model for the self-damping of stranded cables under aeolian vibrations, Journal of Wind Engineering $\&$ Industrial Aerodynamics, 182, 72-86, 2018.

[9] F. Foti, L. Martinelli, Hysteretic behaviour of Stockbridge dampers: modelling and parameter identification, Mathematical Problems in Engineering, Volume 2018, Article ID 8925121, 17 pages, 2018.

[10] CIGRE, EPRI - Electric Research Power Institute, Transmission Line Reference Book: Wind- induced Conductor Motion, EPRI, Palo Alto, USA, 2006.

[11] M. Ervik, et al., Report on aeolian vibrations, Electra, 124, 40-77, 1986.

[12] C. Gazzola, F. Foti, L. Martinelli, F. Perotti, An efficient semi-analytical procedure for assessing aeolian vibrations of overhead transmission lines, In: Advances in Engineering Materials, Structures and Systems: Innovations, Mechanics and Applications - Zingoni (Ed.), Taylor \& Francis Group, London, 37-42, 2019.

[13] C. Gazzola, F. Foti, L. Martinelli, F. Perotti, An Appraisal of Modelling Strategies for Assessing Aeolian Vibrations of Transmission Lines, In: In: Carcaterra A., Paolone A., Graziani G. (eds) Proceedings of XXIV AIMETA Conference 2019 (AIMETA 2019), 1522-1534, 2020.

[14] A. Giaralis, Wavelet based response spectrum compatible synthesis of accelerograms and statistical linearization based analysis of the peak response of inelastic systems, Rice University (Doctoral Dissertation), Houston, Texas, USA, 2008.

[15] P. Hagedorn, Wind-excited vibrations of transmission lines: a comparison of different mathematical models, Mathematical Modelling, 8, 352-358, 1987.

[16] P. Hagedorn, J. Schmidt, N. Nascimento, Stochastic field processes in the mathematical modelling of damped transmission line vibrations, Mathematical Modelling, 8, 359-363, 1987.

[17] C. Hardy, P. Van Dyke, Field observations on wind-induced conductor motions, Journal of Fluid and Structures, 9, 43-60, 1995.

[18] W. Hurewicz, Lectures on ordinary differential equations, 14th edition, Dover Publications, New York, NY, USA, 2014.

[19] J.E. Hurtado, A.H Barbat, Equivalent linearization of the Bouc-Wen hysteretic model, Engineering Structures, 22, 1121-1132, 2000. 
[20] F. Ikhouane, J. Rodellar, J.E. Hurtado, Analytical characterization of hysteresis loops described by the Bouc-Wen model, Mechanics of Advanced Materials and Structures, 13, 463-472, 2006.

[21] F. Ikhouane, V. Manosa, J. Rodellar, Dynamic properties of the hysteretic Bouc-Wen model, Systems and Control Letters, 56, 197-205, 2007.

[22] F. Ikhouane, J.E. Hurtado, J. Rodellar, Variation of the hysteresis loop with the Bouc-Wen model parameters, Nonlinear Dynamics, 48, 361-380, 2007.

[23] M. Ismail, F. Ikhouane, J. Rodellar, The hysteresis Bouc-Wen model, a survey, Archives of Computational Methods in Engineering: State-of-the-Art Reviews, 16, 161-188, 2009.

[24] D.E. Newland, An Introduction to: Random Vibrations, Spectral and Wavelet Analysis, Third Edition, Dover Publications, New York, NY, USA, 2005.

[25] M.P. Paidoussis, S.J. Price, E. De Langre, Fluid-structure interactions: cross-flowinduced instabilities, Cambridge University Press, 2010.

[26] I. Pivovarov, O.G. Vinogradov, One application of Bouc's model for non-linear hysteresis, Journal of Sound and Vibration, 118, 209-216, 1987.

[27] D. Sauter, Modeling the dynamic characteristics of slack wire cables in Stockbridge dampers, Technische Universitat Darmstadt (Dissertation), Darmstadt, Germany, 2003.

[28] D. Sauter, P. Hagedorn, On the hysteresis of wire cables in Stockbridge dampers, International Journal of Non-Linear Mechanics, 37, 1453-1459, 2002.

[29] B.J. Vickery, A.W. Clark, Lift or across-wind response of tapered stackss, Journal of Structural Division (ASCE), 98, 1-20, 1972. 